\title{
Design and analysis of symmetric and compact 2R1T (in-plane 3-DOC) flexurre parallel mechanisms
}

\author{
Guangbo Hao \\ School of Engineering, University College Cork, Cork, Ireland \\ Correspondence to: Guangbo Hao (g.hao@ucc.ie)
}

Received: 30 August 2016 - Revised: 18 December 2016 - Accepted: 30 December 2016 - Published: 10 January 2017

\begin{abstract}
Symmetry is very necessary in flexure mechanisms, which can eliminate parasitic motions, avoid buckling, and minimize thermal and manufacturing sensitivity. This paper proposes two symmetric and compact flexure designs, in-plane 3-DOC (degree of constraint) mechanisms, which are composed of 4 and 6 identical wire beams, respectively. Compared to traditional leaf-beam-based designs, the two present designs have lower stiffness in the primary motion directions, and have smaller stiffness reduction in the parasitic directions. Analytical modelling is conducted to derive the symbolic compliance equations, enabling quick analysis and comparisons of compliances of the two mechanisms. A prototype has been tested statically to compare with analytical models.
\end{abstract}

\section{Introduction}

Flexure mechanisms utilise flexibility/deformation of material to achieve desired functions associated with motion, load and energy, rather than suppress the flexibility (Howell, 2001; Lobontiu, 2002; Howell et al., 2013; Smith, 2003). They offer low cost, high performance, and miniaturization for applications in which traditional mechanisms are not satisfactory since they have no backlash and friction, no wear, no need of lubrication, and reduced number of parts (Howell, 2001; Lobontiu, 2002; Howell et al., 2013; Smith, 2003). Given their advantages, flexure mechanisms have become one of the most popular research areas in mechanisms and robotics over the last two decades. This paper focuses on the design of in-plane 3-DOC (degree of constraint), i.e., outof-plane 2R1T (R: revolute; T: translational), flexure parallel mechanisms. The 3-DOC flexure mechanism can be used as a coordinating measuring machine (Liu et al., 2003), a planar compositional unit of the guiding mechanism of linear actuators (Teo et al., 2015; Awtar and Slocum, 2005; Kim et al., 2013), a compositional unit of the continuum robot (Qiu et al., 2016), or an independent micro-/nano-manipulator (Yu et al., 2004).

Symmetry is very desired in flexure mechanisms, helping eliminate parasitic motions (Awtar and Slocum, 2005), avoid buckling, and minimize thermal and manufacturing sensitiv- ity (Panas and Hopkins, 2015; Hao and Li, 2016). From the point of view of low-power input, avoiding stress concentration and enlarging motion range, a distributed-compliance design is always preferred. There are symmetrical diaphragm in-plane 3-DOC flexure mechanisms used for designing linear guiding mechanisms (Teo et al., 2015; Awtar and Slocum, 2005). The diaphragm flexure is made from a piece of hollow circular plate (plate plane perpendicular to the linear actuation direction). Nevertheless, there may be three issues with the diaphragm design. One is that the actuation stiffness/actuation force is still relatively large due to using the deformation of leaf beams (Teo et al., 2015). The second issue is that the in-plane stiffness of the leaf beam in the plate plane is relatively small since the leaf's thickness in the DOC (degree of constraint) direction is limited by the dimension of the plate (Awtar and Slocum, 2005), and that this DOC stiffness can degrade significantly over the primary motion (Nijenhuis et al., 2015). The final issue is that modelling a leaf beam is extremely complex when considering warping effect and effective modulus to be used (Nijenhuis et al., 2015; Zettl et al., 2004). In order to address the above stiffness issues, References (Hao and Kong, 2014; Merriam and Howell, 2016) have used wire-beam based design to replace the leaf beam for the applications in an $X Y Z$ manipulator and in a cross-axis flexure pivot, respectively. 
Based on the above advances, this paper aims to design and analyse a class of symmetrical and compact in-plane 3DOC flexure mechanisms (only considering purely parallel mechanisms). This paper uses wire beams as the distributedcompliance basic modules to design such flexure mechanisms, which can avoid significant DOC stiffness decrease over the primary motion as caused by the leaf-beam and enable simple modelling using the Young's modulus directly. Analytical expressions of two 3-DOC flexure mechanisms are provided, allowing rapid assessment of various geometric parameters, and performance characteristics of the two flexure mechanisms are compared. The remainder of this paper is organised as follows. Section 2 designs symmetrical and compact in-plane 3-DOC flexure mechanisms followed by compliance modelling and analysis in Sect. 3. In Sect. 4, a prototype is fabricated and tested, and actuation isolation is discussed. Finally, conclusions are drawn in Sect. 5.

\section{Design Considerations}

Throughout this paper, all wire beams employed for the inplane flexure mechanism are specified to be identical with a length of $L$ and uniform square cross-sections (with a thickness $T$ ) for convenience. We set up a global coordinate system $(O-X Y Z)$ at the body centre of the motion stage of a flexure mechanism, where the $Z$ axis is perpendicular to the in-plane 3-DOC mechanism.

The exactly-constrained in-plane 3-DOC is a 3-wire-beam parallel mechanism with a general arrangement based on the complimentary theory (Fig. 1), which can be easily designed using existing methods such as FACT or other screw-theorybased methods (Hopkins and Culpepper, 2010a, b; Su and Hafez, 2010; Su et al., 2009; Yu et al., 2011). To guarantee an in-plane 3-DOC mechanism, the following conditions in plane should be met:

1. To avoid translational motion in plane: not all beams can be parallel or co-linear, i.e., there should be intersection among beams;

2. To avoid rotational motion in plane: if there is intersection among beams (not infinitely far), there should be more than one intersection points and the distance between two intersection points of beams should be large enough.

Nevertheless, in order to design a symmetrical flexure mechanism, the number of beams should be even at least with the minimal number of 4 where the wire beams should equally distribute about at least one axis ( $X$ or $Y$ axis). Moreover, in order to obtain the most compact configuration, all beams should uniformly distribute around the $Z$ axis, i.e., the intersection points of all wire beams form the vertices of a regular polygon. Figure 2 shows the two symmetric and compact designs, composed of 4 and 6 wire beams, respectively, which are the focus of this paper. Other symmetrical and compact

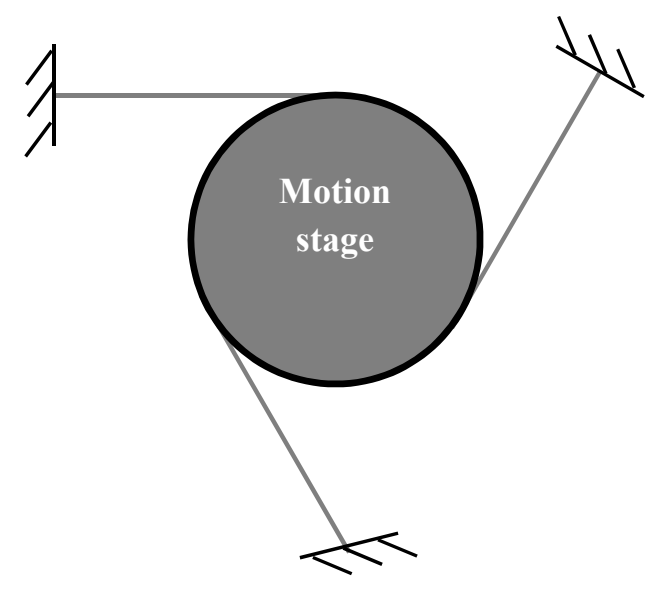

Figure 1. Exactly-constrained non-symmetric design of an in-plane 3-DOC mechanism.

in-plane 3-DOC mechanisms with more beams can be designed in a similar way.

Note that the design method of this section in proposing an in-plane 3-DOC flexure mechanism has been reported in Hao (2017) in a similar way. In Hao (2017), the research work is on the design and analysis of a symmetric 1-DOF (degree of freedom) translational joint for guiding linear actuators, the symmetric in-plane 3-DOC mechanism composed of 4 or 6 wire beams is presented only as a compositional unit of the 1-DOF mechanism. However, in Hao (2017) there was no information on the design of most compact mechanisms with any even number of wire beams, there was no analytical modelling done for any in-plane 3-DOC mechanism; there was no parameter study on the quick performance assessment of any 3-DOC mechanism, there was no characteristic comparison of two types of flexure mechanisms with 4 and 6 wire beams, respectively, and there was no experimental testing for any 3-DOC mechanism. This paper will carry out the above undone work for two symmetric and compact 3-DOC mechanisms (Fig. 2).

\section{Compliance Modelling and Analysis}

This section proposes an analytical method (linear method) to model the two symmetrical and compact flexure mechanisms (Fig. 2), which enables a quick compliance analysis.

\subsection{Modelling Method}

The analytical modelling method is detailed in the following steps.

1. Normalise geometrical dimension by the mechanism's characteristic length $L_{\mathrm{c}}\left(L_{\mathrm{c}}=L\right.$ in this paper, which is the beam length), and normalise the force by $E I / L^{2}$, and moment by $E I / L$ ( $E$ : Young's modulus; $I=$ 


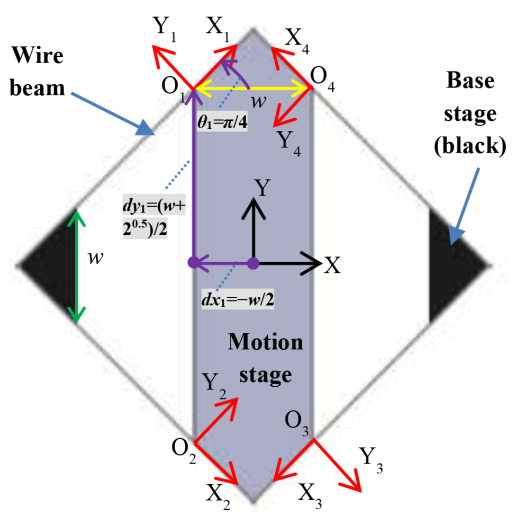

(a) Symmetric and compact 4-beam in-plane 3-DOC mechanism

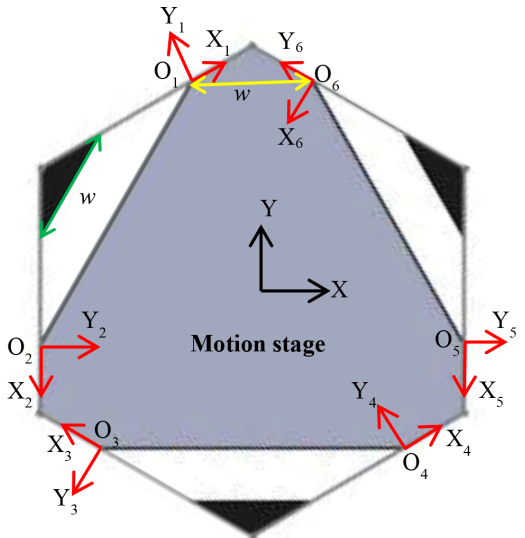

(b) Symmetric and compact 6-beam in-plane 3-DOC mechanism

Figure 2. Two symmetrical and compact 3-DOC mechanisms ( $w$ is the distance between two beams' tips).

Table 1. Geometrical parameter relationships.

\begin{tabular}{|c|c|c|c|c|c|c|}
\hline & $\begin{array}{l}\text { Beam 1's local } \\
\text { coordinate system } \\
\text { position parame- } \\
\text { ters w.r.t the global } \\
\text { coordinate system }\end{array}$ & $\begin{array}{l}\text { Beam 2's local } \\
\text { coordinate system } \\
\text { position parame- } \\
\text { ters w.r.t the global } \\
\text { coordinate system }\end{array}$ & $\begin{array}{l}\text { Beam } 3 \text { local's coor- } \\
\text { dinate system posi- } \\
\text { tion parameters w.r.t } \\
\text { the global coordi- } \\
\text { nate system }\end{array}$ & $\begin{array}{l}\text { Beam 4's local coor- } \\
\text { dinate system posi- } \\
\text { tion parameters w.r.t } \\
\text { the global coordi- } \\
\text { nate system }\end{array}$ & $\begin{array}{l}\text { Beam 5's local } \\
\text { coordinate system } \\
\text { position parame- } \\
\text { ters w.r.t the global } \\
\text { coordinate system }\end{array}$ & $\begin{array}{l}\text { Beam 6's local } \\
\text { coordinate system } \\
\text { position parame- } \\
\text { ters w.r.t the global } \\
\text { coordinate system }\end{array}$ \\
\hline $\begin{array}{l}\text { 4-beam } \\
\text { mechanism }\end{array}$ & $\begin{array}{l}\mathrm{d} x_{1}=-w / 2 \\
\mathrm{~d} y_{1}=\frac{(w+\sqrt{2})}{2} \\
\theta_{1}=\pi / 4\end{array}$ & $\begin{array}{l}\mathrm{d} x_{2}=-w / 2 \\
\mathrm{~d} y_{2}=-\frac{(w+\sqrt{2})}{2} \\
\theta_{2}=-\pi / 4\end{array}$ & $\begin{array}{l}\mathrm{d} x_{3}=w / 2 \\
\mathrm{~d} y_{3}=-\frac{(w+\sqrt{2})}{2} \\
\theta_{3}=-3 \pi / 4\end{array}$ & $\begin{array}{l}\mathrm{d} x_{4}=w / 2 \\
\mathrm{~d} y_{4}=\frac{(w+\sqrt{2})}{2} \\
\theta_{4}=3 \pi / 4\end{array}$ & NA & NA \\
\hline $\begin{array}{l}\text { 6-beam } \\
\text { mechanism }\end{array}$ & $\begin{array}{l}\mathrm{d} x_{1}=-w / 2 \\
\mathrm{~d} y_{1}=\left(1+\frac{\sqrt{3} w}{2}\right) \\
\theta_{1}=\pi / 6\end{array}$ & $\begin{array}{l}\mathrm{d} x_{2}=-\left(\frac{\sqrt{3}}{2}+w\right) \\
\mathrm{d} y_{2}=-\frac{1}{2} \\
\theta_{2}=3 \pi / 2\end{array}$ & $\begin{array}{l}\mathrm{d} x_{3}=-\left(\frac{\sqrt{3}}{2}+\frac{w}{2}\right) \\
\mathrm{d} y_{3}=-\left(\frac{1}{2}+\frac{\sqrt{3} w}{2}\right) \\
\theta_{3}=5 \pi / 6\end{array}$ & $\begin{array}{l}\mathrm{d} x_{4}=\left(\frac{\sqrt{3}}{2}+\frac{w}{2}\right) \\
\mathrm{d} y_{4}=-\left(\frac{1}{2}+\frac{\sqrt{3} w}{2}\right) \\
\theta_{4}=\pi / 6\end{array}$ & $\begin{array}{l}\mathrm{d} x_{5}=\left(\frac{\sqrt{3}}{2}+w\right) \\
\mathrm{d} y_{5}=-\frac{1}{2} \\
\theta_{5}=3 \pi / 2\end{array}$ & $\begin{array}{l}\mathrm{d} x_{6}=w / 2 \\
\mathrm{~d} y_{6}=\left(1+\frac{\sqrt{3} w}{2}\right) \\
\theta_{6}=5 \pi / 6\end{array}$ \\
\hline
\end{tabular}

$T^{4} / 12$ : second moment of inertia of square crosssection areas) with all normalised parameters represented by corresponding lower-case symbols (Hao and Kong, 2013).

2. Establish a local coordinate system $\left(O_{i}-X_{i} Y_{i} Z_{i}\right)$ at the mobile tip (centre) of each wire beam (Fig. 2). The stiffness matrix of each wire beam with regard to the local coordinate system is expressed as

$$
\mathbf{K}=\left[\begin{array}{cccccc}
d & 0 & 0 & 0 & 0 & 0 \\
0 & 12 & 0 & 0 & 0 & -6 \\
0 & 0 & 12 & 0 & 6 & 0 \\
0 & 0 & 0 & \delta & 0 & 0 \\
0 & 0 & 6 & 0 & 4 & 0 \\
0 & -4 & 0 & 0 & 0 & 4
\end{array}\right]
$$

with $d=12 / t^{2}$ and $\delta=G J /(E I)=1.69 G / E$ in which $J=2.25 T^{4} / 16$ is the torsional constant considering warping (ignoring warping constraint) of square cross sections, and $G$ is the shear modulus (Chen and Bai, 2016).
3. Determine the position parameters of each local coordinate system with regard to the global coordinate system, which are associated with a rotation variable $\theta_{i}$ and two translation variables $\mathrm{d} x_{i}$ and $\mathrm{d} y_{i}$. Table 1 lists all position parameters for the two designs (Fig. 2).

4. Derive the transformation matrix for each wire beam (Hao and Kong, 2013) and then obtain the mechanism's stiffness matrix:

$$
\begin{aligned}
& \mathbf{T R}_{i}=\left[\begin{array}{cccccc}
\cos \left(-\theta_{i}\right) & -\sin \left(-\theta_{i}\right) & 0 & 0 & 0 & 0 \\
\sin \left(-\theta_{i}\right) & \cos \left(-\theta_{i}\right) & 0 & 0 & 0 & 0 \\
0 & 0 & 1 & 0 & 0 & 0 \\
0 & 0 & 0 & \cos \left(-\theta_{i}\right) & -\sin \left(-\theta_{i}\right) & 0 \\
0 & 0 & 0 & \sin \left(-\theta_{i}\right) & \cos \left(-\theta_{i}\right) & 0 \\
0 & 0 & 0 & 0 & 0 & 1
\end{array}\right] \\
& {\left[\begin{array}{cccccc}
1 & 0 & 0 & 0 & 0 & -\mathrm{d} y_{i} \\
0 & 1 & 0 & 0 & 0 & \mathrm{~d} x_{i} \\
0 & 0 & 1 & \mathrm{~d} y_{i} & -\mathrm{d} x_{i} & 0 \\
0 & 0 & 0 & 1 & 0 & 0 \\
0 & 0 & 0 & 0 & 1 & 0 \\
0 & 0 & 0 & 0 & 0 & 1
\end{array}\right]} \\
& =\left[\begin{array}{cc}
\mathbf{R}\left(-\theta_{i}\right)_{3 \times 3} & \mathbf{R}\left(-\theta_{i}\right)_{3 \times 3} \cdot \mathbf{D}\left(\mathrm{d} x_{i}, \mathrm{~d} y_{i}\right)_{3 \times 3} \\
\mathbf{0}_{3 \times 3} & \mathbf{R}\left(-\theta_{i}\right)_{3 \times 3}
\end{array}\right]_{6 \times 6}
\end{aligned}
$$

which transforms the local stiffness matrix of each beam to a stiffness matrix with regard to the origin of the 
Table 2. Characteristic comparisons of the two mechanisms.

\begin{tabular}{lllll}
\hline$c_{11}=c_{22}$ (DOC) & $c_{33}$ (DOF) & $c_{44}=c_{55}$ (DOF) & $c_{66}$ (DOC) & Dimension \\
\hline $\begin{array}{l}\text { 6-beam is smaller, which } \\
\text { is better }\end{array}$ & $\begin{array}{l}\text { 4-beam is larger, which } \\
\text { is better }\end{array}$ & $\begin{array}{l}\text { 4-beam is larger, which } \\
\text { is better }\end{array}$ & $\begin{array}{l}\text { 6-beam is smaller, which } \\
\text { is better }\end{array}$ & $\begin{array}{l}\text { 4-beam is smaller, which } \\
\text { is better }\end{array}$ \\
\hline
\end{tabular}

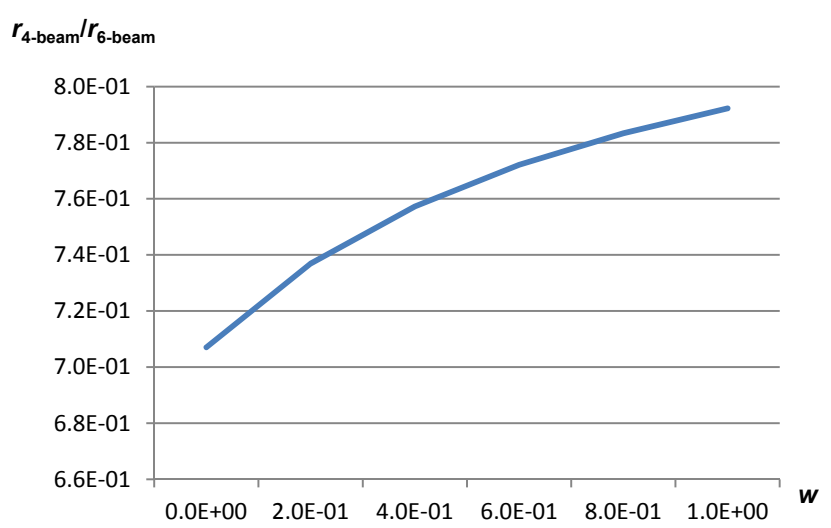

Figure 3. Dimension comparison of two symmetric mechanisms.

global coordinate system. In such a way, we can obtain the stiffness matrix of the symmetrical flexure mechanism (parallel one) at the centre of the global coordinate system as:

$$
\mathbf{K}_{\mathrm{FM}}=\Sigma\left[\left(\mathbf{T} \mathbf{R}_{i}\right)^{\mathrm{T}} \cdot \mathbf{K} \cdot \mathbf{T} \mathbf{R}_{i}\right]
$$

5. Obtain the compliance matrix of the symmetrical flexure mechanism: $\mathbf{C}_{\mathrm{FM}}=\left(\mathbf{K}_{\mathrm{FM}}\right)^{-1}$, and then double check if entries of the compliance matrix meet the following two criteria:

a. The compliance matrix should be a diagonal matrix for ensuring symmetry as shown below

$$
\mathbf{C}_{\mathrm{FM}}=\left[\begin{array}{cccccc}
c_{11} & 0 & 0 & 0 & 0 & 0 \\
0 & c_{22} & 0 & 0 & 0 & 0 \\
0 & 0 & {\left[c_{33}\right]} & 0 & 0 & 0 \\
0 & 0 & 0 & {\left[c_{44}\right]} & 0 & 0 \\
0 & 0 & 0 & 0 & {\left[c_{55}\right]} & 0 \\
0 & 0 & 0 & 0 & 0 & c_{66}
\end{array}\right]
$$

where $c_{11},\left(c_{22}\right.$ or $\left.c_{33}\right)$ relates the translational displacement along the $X$ axis ( $Y$ or $Z$ axis) and the force along the same axis; $c_{44},\left(c_{55}\right.$ or $\left.c_{66}\right)$ relates the rotational displacement around the $X$ axis ( $Y$ or $Z$ axis) and the moment around the same axis.

b. The diagonal entries corresponding to the three DOC, $c_{11}, c_{22}$, and $c_{66}$, should be much smaller than the other diagonal entries associated with the three DOF (degree of freedom), $c_{33}, c_{44}$, and $c_{55}$. The DOF entry is usually $10^{2}$ times smaller than the DOC entry (Hao and Kong, 2013).
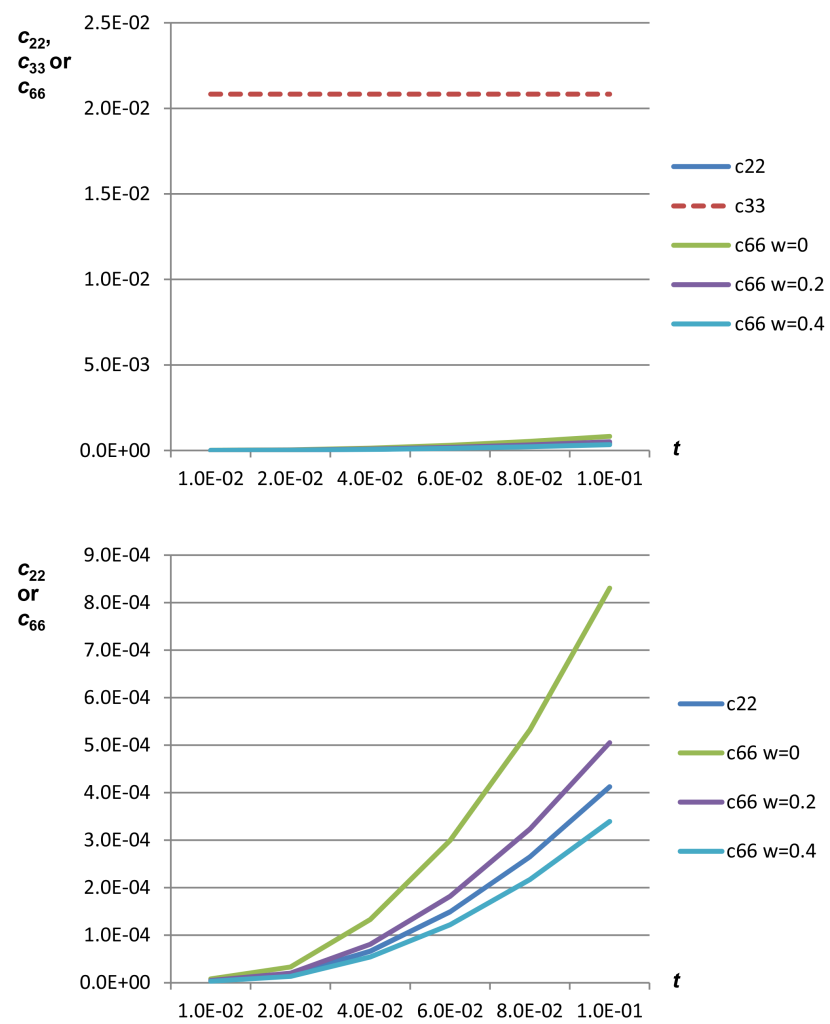

(a) Compliance affected by $t$

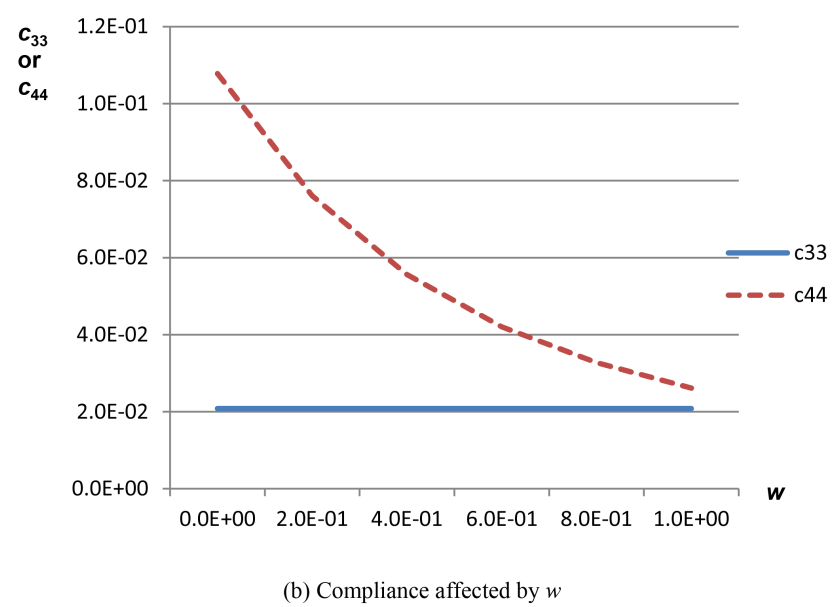

Figure 4. Compliance comparisons of symmetric 4-beam mechanism. 
Table 3. FEA comparisons for compliances.

\begin{tabular}{|c|c|c|c|c|c|c|c|c|}
\hline \multirow[b]{2}{*}{$\begin{array}{l}\text { 4-beam } \\
\text { mechanism }\end{array}$} & \multicolumn{2}{|c|}{$c_{11}=c_{22}$} & \multicolumn{2}{|c|}{$c_{33}$} & \multicolumn{2}{|c|}{$c_{44}=c_{55}$} & \multicolumn{2}{|c|}{$c_{66}$} \\
\hline & $\begin{array}{l}\text { Analytical: } \\
4.62 \times 10^{-5}\end{array}$ & $\begin{array}{l}\text { FEA: } \\
4.80 \times 10^{-5}\end{array}$ & $\begin{array}{l}\text { Analytical: } \\
2.08 \times 10^{-2}\end{array}$ & $\begin{array}{l}\text { FEA: } \\
2.15 \times 10^{-2}\end{array}$ & $\begin{array}{l}\text { Analytical: } \\
5.01 \times 10^{-2}\end{array}$ & $\begin{array}{l}\text { FEA: } \\
5.24 \times 10^{-2}\end{array}$ & $\begin{array}{l}\text { Analytical: } \\
3.33 \times 10^{-5}\end{array}$ & $\begin{array}{l}\text { FEA: } \\
3.48 \times 10^{-5}\end{array}$ \\
\hline & \multicolumn{2}{|c|}{ Difference: $3.75 \%$} & \multicolumn{2}{|c|}{ Difference: $3.25 \%$} & \multicolumn{2}{|c|}{ Difference: $4.37 \%$} & \multicolumn{2}{|c|}{ Difference: $4.31 \%$} \\
\hline \multirow[t]{2}{*}{$\begin{array}{l}\text { 6-beam } \\
\text { mechanism }\end{array}$} & $\begin{array}{l}\text { Analytical: } \\
3.08 \times 10^{-5}\end{array}$ & $\begin{array}{l}\text { FEA: } \\
3.19 \times 10^{-5}\end{array}$ & $\begin{array}{l}\text { Analytical: } \\
1.39 \times 10^{-2}\end{array}$ & $\begin{array}{l}\text { FEA: } \\
1.44 \times 10^{-2}\end{array}$ & $\begin{array}{l}\text { Analytical: } \\
1.44 \times 10^{-2}\end{array}$ & $\begin{array}{l}\text { FEA: } \\
1.50 \times 10^{-2}\end{array}$ & $\begin{array}{l}\text { Analytical: } \\
4.31 \times 10^{-6}\end{array}$ & $\begin{array}{l}\text { FEA: } \\
4.51 \times 10^{-6}\end{array}$ \\
\hline & \multicolumn{2}{|c|}{ Difference: $3.45 \%$} & \multicolumn{2}{|c|}{ Difference: $3.47 \%$} & \multicolumn{2}{|c|}{ Difference: $4.00 \%$} & \multicolumn{2}{|c|}{ Difference: $4.43 \%$} \\
\hline
\end{tabular}

\subsection{Compliance Analysis}

Based on the procedure in Sect. 3.1, a diagonal compliance matrix for the 4-beam or 6-beam mechanism can be obtained, and its entries in the diagonal can be symbolically expressed by parameters $(\delta, d, w)$ as shown in

$$
\begin{aligned}
& \mathbf{C}_{\mathrm{FM}-4}=\operatorname{diag}\left(\frac{1}{2(d+12)}, \frac{1}{2(d+12)}, \frac{1}{48},\right. \\
& \frac{1}{2\left(6 w^{2}+6 \sqrt{2} w+\delta+4\right)}, \frac{1}{2\left(6 w^{2}+6 \sqrt{2} w+\delta+4\right)}, \\
& \left.\frac{1}{2 \mathrm{~d} w^{2}+2 \sqrt{2} \mathrm{~d} w+d+4}\right) \\
& \mathbf{C}_{\mathrm{FM}-6}=\operatorname{diag}\left(\frac{1}{3(d+12)}, \frac{1}{3(d+12)}, \frac{1}{72},\right. \\
& \frac{1}{3\left(12 w^{2}+12 \sqrt{3} w+\delta+10\right)}, \\
& \frac{1}{3\left(12 w^{2}+12 \sqrt{3} w+\delta+10\right)}, \\
& \left.\frac{1}{3\left(4 \mathrm{~d} w^{2}+4 \sqrt{3} \mathrm{~d} w+3 d+4\right)}\right)
\end{aligned}
$$

where $c_{33}$ is constant for both symmetric flexure mechanisms. The $c_{33}$ value for the 4-beam mechanism is 1.5 times larger than that for the 6-beam mechanism, which means the 4-beam mechanism requires less actuation force. For any mechanism, $c_{11}$, equal to $c_{22}$, decreases with the increase of $d ; c_{44}$, equal to $c_{55}$, decreases with the increase of $w$ or $\delta$; and $c_{66}$ decreases with the increase of $d$ or $w$. Since $d$ is a very large value (inversely proportional to square of the normalised beam thickness) and is included in the denominator of compliance entries of $c_{11}, c_{22}$ and $c_{66}$, the three in-plane motions are effectively constrained. Usually, aluminium alloy $6061 \mathrm{~T} 651$ with $E=69 \mathrm{GPa}$ and $G=26 \mathrm{GPa}$ is used for fabricating flexure beams, so $\delta=0.64$ are adopted in the following analysis.
If $w=0$, Eqs. (5a) and (5b) reduce to

$$
\begin{gathered}
\mathbf{C}_{\mathrm{FM}-4}=\operatorname{diag}\left(\frac{1}{2(d+12)}, \frac{1}{2(d+12)}, \frac{1}{48}, \frac{1}{2(\delta+4)},\right. \\
\left.\frac{1}{2(\delta+4)}, \frac{1}{d+4}\right) \\
\mathbf{C}_{\mathrm{FM}-6}=\operatorname{diag}\left(\frac{1}{3(d+12)}, \frac{1}{3(d+12)}, \frac{1}{72}, \frac{1}{3(\delta+10)},\right. \\
\left.\frac{1}{3(\delta+10)}, \frac{1}{3(3 d+4)}\right)
\end{gathered}
$$

The distance between the motion stage centre and vertex of a regular polygon is $r_{4 \text {-beam }}=\sqrt{2} / 2+w$ for the 4-beam mechanism, or $r_{6 \text {-beam }}=1+2 \sqrt{3} w / 3$ for the 6-beam mechanism. If $w$ is the same for the 4-beam and 6-beam flexure mechanisms, the dimension ratio $\left(r_{4-\text { beam }} / r_{6 \text {-beam }}\right)$ between the two mechanisms is plotted in Fig. 3. It can be seen that the dimension of the 4-beam mechanism is smaller than that of the 6-beam mechanism. The dimension ratio increases with the increase of $w$.

Figure 4 shows the compliance comparisons in the 4-beam mechanism. It is observed that $c_{33}$ is much larger than $c_{22}$ or $c_{66}$ as expected but it is comparable with $c_{44}$. Figure 5 shows the similar conclusion for the 6-beam mechanism. The additional compliance comparisons between the 4-beam and 6beam mechanisms are illustrated in Fig. 6 . It can be seen that $c_{22}, c_{44}$, and $c_{66}$ in the 4-beam mechanism are larger than those in the 6-beam mechanism, respectively. The smaller the compliance entry associated with the DOC and the larger the compliance entry associated with the DOF, the better the mechanism is. Table 2 summarises the characteristic comparisons of the two mechanisms.

Linear FEA simulations using Comsol with fine and free tetrahedral meshing for the two mechanism were carried out for the case with $w=2^{0.5} / 3$ and $t=1 / 30$. Compliance comparisons between FEA model and the analytical linear model are shown in Table 3. It is observed that both models have good agreement with less than $5 \%$ difference and FEA results are slightly larger as predicted. 

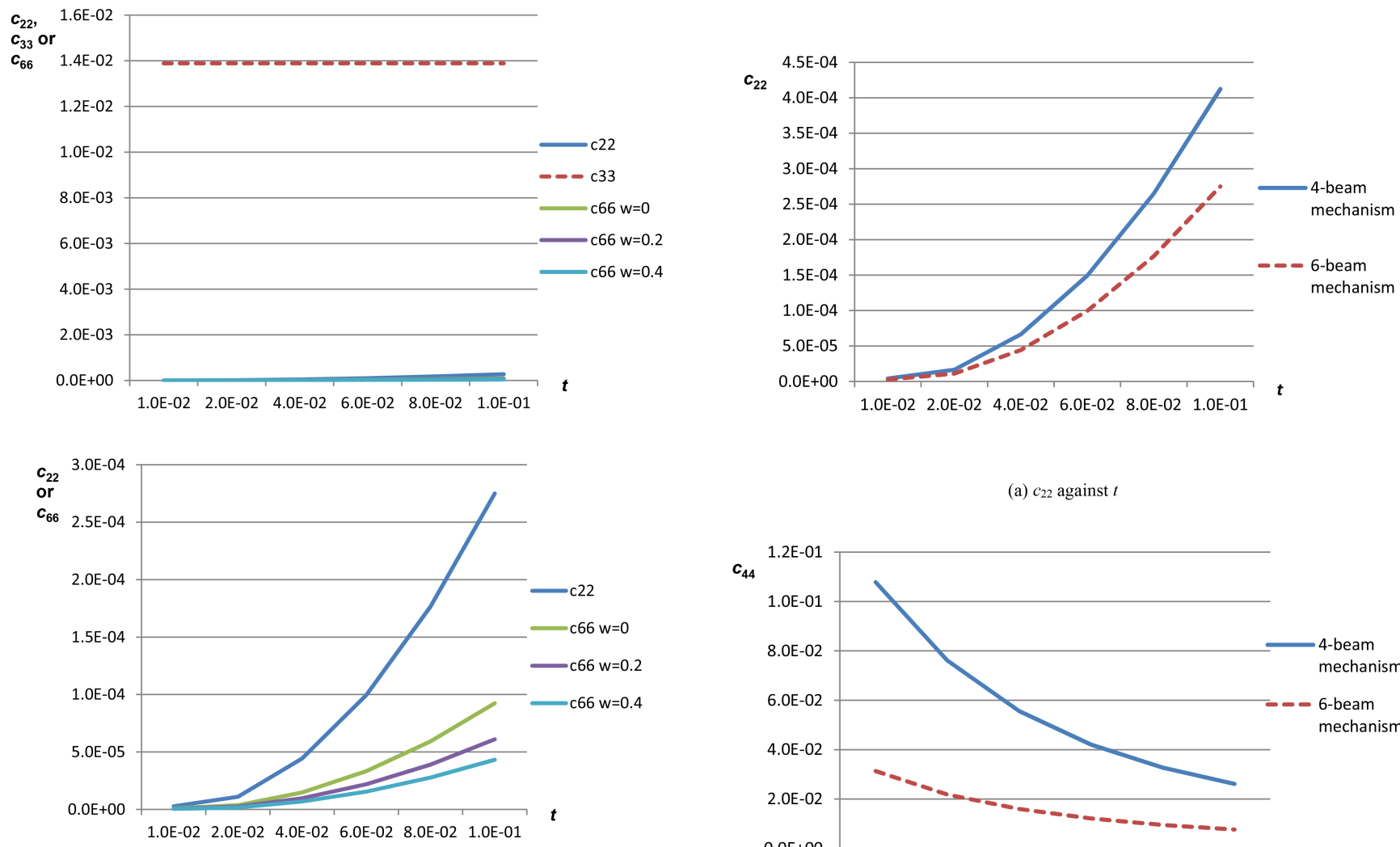

(a) Compliance affected by $t$

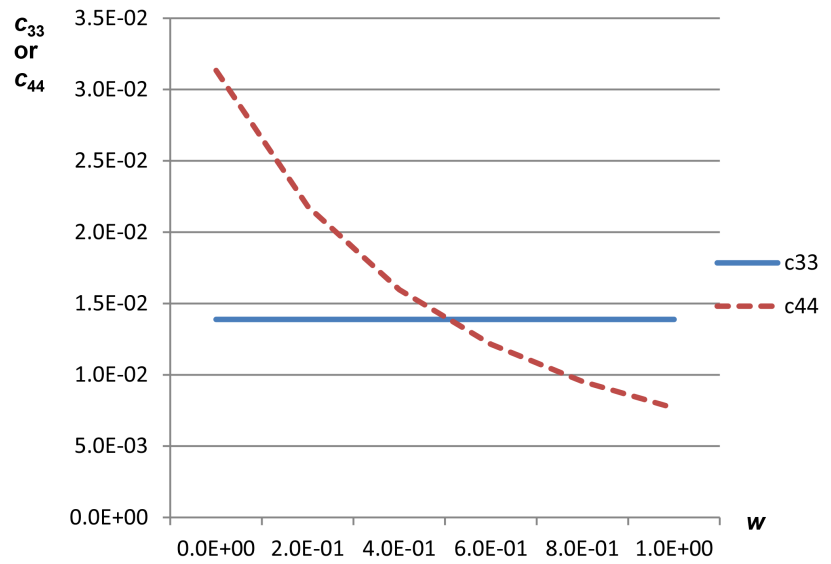

(b) Compliance affected by $w$

Figure 5. Compliance comparisons of symmetric 6-beam mechanism. 


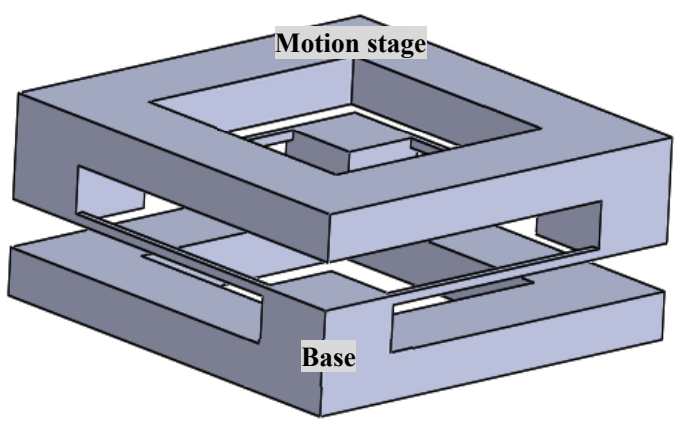

(a) CAD design

Figure 7. A practical 2R1T design without actuation isolation.

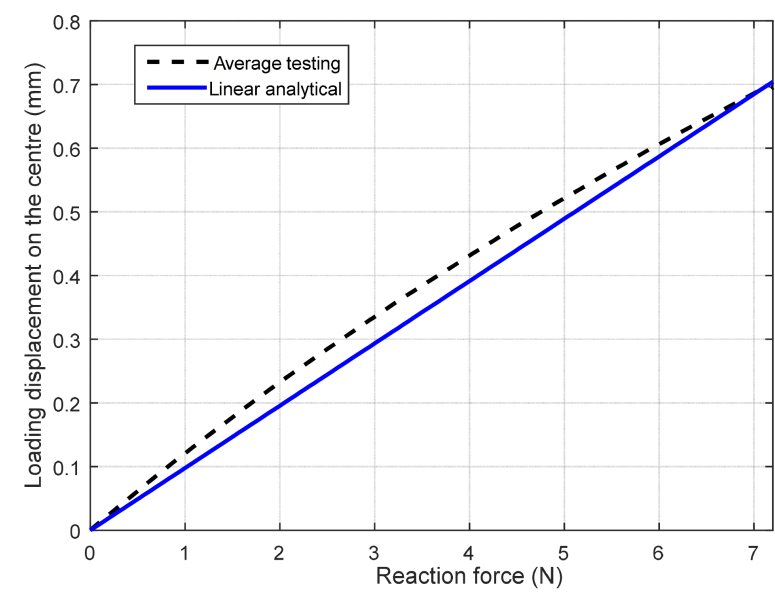

(a) Loading scenario 1: loading displacement on the centre

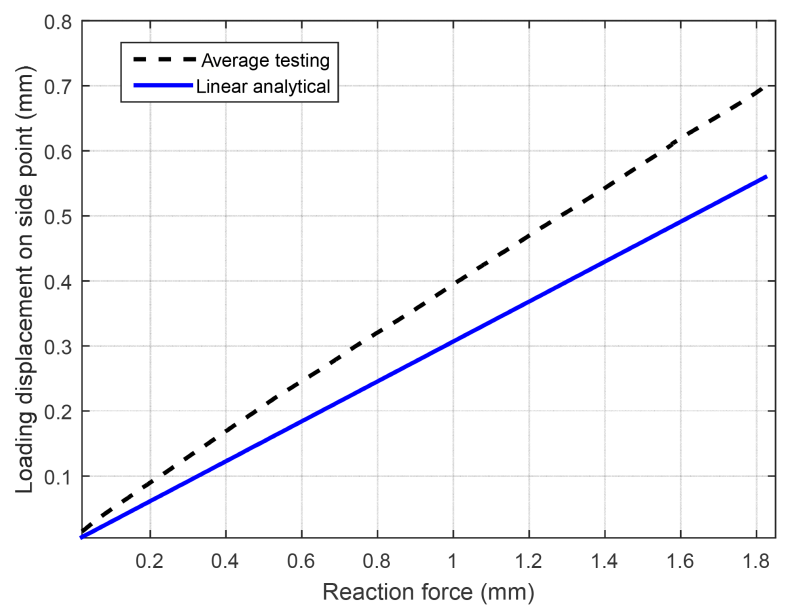

(b) Loading scenario 2: loading displacement on the side

Figure 8. Testing results of the in-plane 3-DOC mechanism in the DOF directions.

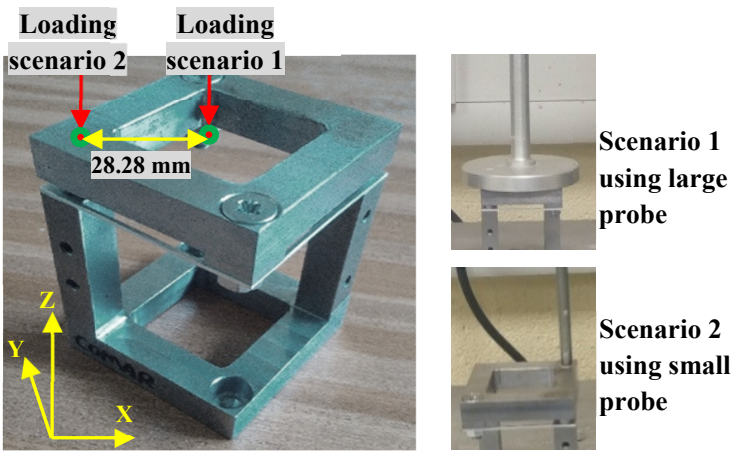

(b) Prototype and testing

\section{Testing and discussions}

The proposed symmetric and compact flexure mechanisms (Fig. 2) can be used as independent in-plane 3-DOC (tiptilt-piston) mechanisms. Figure 7 shows a practical 4-beam design using non-contact (such as magnetic) actuation for such an application when $w=2^{0.5} / 3$ and $t=1 / 30$ for $L=$ $30 \mathrm{~mm}$.

The prototype for such a design has also been fabricated by $\mathrm{CNC}$ milling machining (Fig. 7b), and the testing results comparing to the analytical models are shown in Fig. 8. A single-axis loading bi-directional tester (TA.Hd plus texture analyser) was used for conducting the testing, which can apply a series of specific displacements on the mechanism and record the corresponding reaction forces automatically. A load cell of $5 \mathrm{~kg}$ with force resolution of $0.1 \mathrm{~g}$ and a loading displacement increment of $0.05 \mathrm{~mm}$ were selected, and the testing speed was controlled very lowly at $0.01 \mathrm{~m} \mathrm{~s}^{-1}$ to eliminate any dynamic response. We implemented two loading scenarios as indicated in Fig. $7 \mathrm{~b}$. The first scenario is to exert displacement loading on the centre of the motion stage (using a large probe) and obtain the corresponding reaction force on the same centre, which is to test the compliance in the $Z$ axis with regard to the centre as shown in Fig. 8a. The second scenario is to exert displacement loading on the side ( $28.28 \mathrm{~mm}$ off the centre, using a very small probe) and obtain the corresponding reaction force on the same point, which is to test the resulting displacements caused by the force along the $Z$ axis (located at the centre) and the moment (rotation) about the $Y$ axis (Fig. 8b). The actual analytical displacement $Z_{\text {off }}$ (in $\mathrm{mm}$ ) on the side point can be calculated below against the associated reaction force $F_{\text {off }}$ (in N):

$$
\begin{aligned}
Z_{\text {off }} & =c_{33} \times F_{\text {off }} /\left(E I / L^{2}\right) \times L+c_{55} \\
& \times\left(F_{\text {off }} \times 28.28\right) /(E I / L) \times 28.28(\mathrm{~mm})
\end{aligned}
$$

From the plotted result comparisons in Fig. 8, we can observe: that the loading scenario 1 has a better match between the testing and analytical models than the scenarios 2 ; that 


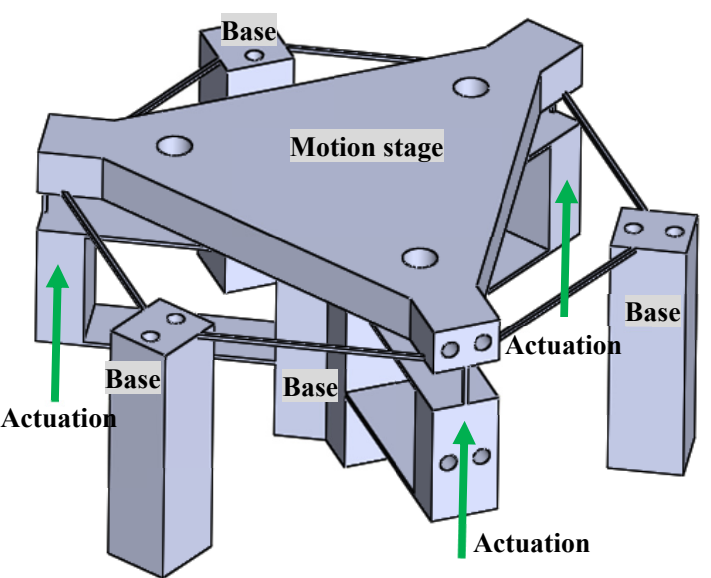

(a) 2R1T positioning stage

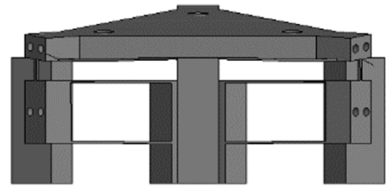

(b) FEA rotational result by one actuation

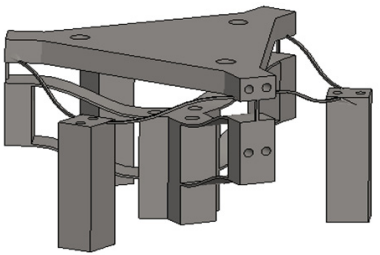

(c) FEA translational result by three equal actuations

Figure 9. A 2R1T positioning stage with actuation isolation.

the scenario 2 shows a linear relation in testing as opposed to the slightly-nonlinear relation in testing in the scenario 1 ; and that the testing displacement is generally larger than analytical one in both scenarios. The deviation between the two models in Fig. 8 can be from the assumptions of small deformation of flexure elements and of ideal-rigid-body of nonflexure elements in the linear modelling (Sect. 3).

Contact actuation using linear PZT or VC actuators are common in high-precision positioning control, therefore, a new design with actuation isolation (linear actuators are guided to tolerate transverse motion/load) should be considered as shown in Fig. 9. Here, three flexure legs are added to the $2 \mathrm{R} 1 \mathrm{~T}$ 6-beam motion stage in parallel where each one consists of an actuated translational joint (parallelogram mechanism for instance) and a passive (5-DOF) wire beam with its axis direction perpendicular to the plane of the motion stage. The wire beam in each added leg can offer the rotational and slight transverse motions to decouple the motion between the 2R1T mechanism and the actuation. The new design with actuation isolation is generated based on the following design rule: the addition of any number of 6-DOF legs to an original $n$-DOF (parallel) mechanism results in a parallel mechanism with the same number of DOF and more legs.

\section{Conclusions}

Two symmetrical and compact 2R1T (in-plane 3-DOC) flexure mechanisms have been designed in this paper for promoting better performances. The two designs are composed of 4 and 6 identical wire beams, respectively, which have lower stiffness in the DOF directions, with smaller stiffness reduction in DOC directions. Analytical modelling has been conducted to analyse and compare compliances of the two flexure mechanisms. A prototype has been fabricated with testing results compared with the analytical models.

It is noted that other symmetrical and compact in-plane 3DOC mechanisms with more beams (even number) can be designed and modelled using the method in this paper. Further nonlinear analysis is to be investigated.

Acknowledgements. The author would like to sincerely thank Tim Power, Mike O'Shea at University College Cork for their excellent fabrication of the prototype. Haiyang Li and George Joseph are also appreciated for their kind help in producing the testing results.

Edited by: D. Pisla

Reviewed by: three anonymous referees

\section{References}

Awtar, S. and Slocum, A. H.: Design of Flexure Stages based on a Symmetric Diaphragm Flexure, Proceedings of ASPE 2005 Annual Meeting, Norfolk, VA, USA, Paper No. 1803, 2005.

Chen, G. and Bai, R.: Modeling Large Spatial Deflections of Slender Bisymmetric Beams in Compliant Mechanisms Using Chained Spatial-Beam Constraint Model, Journal of Mechanisms and Robotics, 8, 041011, doi:10.1115/1.4032632, 2016.

Hao, G.: Determinate design and analytical analysis of a class of symmetrical flexure guiding mechanisms for linear actuators, ASME J. Mech. Design, 139, 012301, doi:10.1115/1.4034579, 2017.

Hao, G. and Kong, X.: A Normalization-Based Approach to the Mobility Analysis of Spatial Compliant Multi-Beam Modulus, Mech. Mach. Theory, 59, 1-19, 2013.

Hao, G. and Kong, X.: Nonlinear Analytical Modelling and Characteristic Analysis of Symmetrical-Beam Based Composite Compliant Parallel Modulus for Planar Motion, Mech. Mach. Theory, 77, 122-147, 2014. 
Hao, G. and Li, H.: Extended Static Modeling and Analysis of Compliant Compound Parallelogram Mechanisms Considering the Initial Internal Axial Force, ASME J. Mechanisms Robotics, 8, 041008, doi:10.1115/1.4032592, 2016.

Hopkins, J. B. and Culpepper, M. L.: Synthesis of Multi-degree of Freedom, Parallel Flexure System Concepts via Freedom and Constraint Topology (FACT) - Part I: Principles, Precis. Eng., 34, 259-270, 2010a.

Hopkins, J. B. and Culpepper, M. L.: Synthesis of Multi-degree of Freedom, Parallel Flexure System Concepts via Freedom and Constraint Topology (FACT) - Part II: Practice, Precis. Eng., 34, 271-278, 2010b.

Howell, L. L.: Compliant Mechanisms, Wiley, New York, 2001.

Howell, L. L., Magleby, S. P., and Olsen, B. M.: Handbook of Compliant Mechanisms, Wiley, New York, 2013.

Kim, C., Song, M.-G, Kim, Y. J., Park, N.-C., Park, K.-S., Park, Y.-P., Shin, K. S., Kim, J. G., and Lee, G. S.: Design of an autofocusing actuator with a flexure-based compliant mechanism for mobile imaging devices, Microsyst. Technol., 19, 1633-1644, 2013

Liu, D., Che, R., and Li, Z.: Research on the theory and the virtual prototype of 3-DOF parallel-link coordinating measuring machine, IEEE T. Instrum. Meas., 52, 119-125, 2003.

Lobontiu, N.: Compliant Mechanisms: Design of Flexure Hinges, CRC Press, Boca Raton, 2002.

Merriam, E. G. and Howell, L. L.: Lattice flexures: Geometries for stiffness reduction of blade flexures, Precis. Eng., 45, 160-167, 2016.

Nijenhuis, M., Meijaard, J. P., Herder, J., Awtar, S., and Brouwer, D. M.: An Analytical formulation for the lateral support stiffness of a spatial flexure strip, Proceedings of the ASME 2015 International Design Engineering Technical Conferences \& Computers and Information in Engineering Conference (IDETC/CIE 2015), 2-5 August 2015, Boston, Massachusetts, USA, DETC201546591, 2015.
Panas, R. M. and Hopkins, J. B.: Eliminating Underconstraint in Double Parallelogram Flexure Mechanisms, ASME J. Mech. Design, 137, 092301, doi:10.1115/1.4030773, 2015.

Qiu, C., Qi, P., Liu, H., Althoefer, K., and Dai, J. S.: SixDimensional Compliance Analysis and Validation of Orthoplanar Springs, ASME J. Mech. Design, 138, 042301, doi:10.1115/1.4032580, 2016.

Smith, S. T.: Flexures: Elements of Elastic Mechanisms, Taylor and Francis, London, 2003.

Su, H.-J. and Hafez, T.: Realizing Orthogonal Motions with Wire Flexures Connected in Parallel, J. Mech. Design, 132, 121002, doi:10.1115/1.4002837, 2010.

Su, H.-J., Denis, V. D., and Judy, M. V.: A Screw Theory Approach for the Conceptual Design of Flexible Joints for Compliant Mechanisms, Journal of Mechanisms and Robotics, 1, 041009, doi:10.1115/1.3211024, 2009.

Teo, T., Yang, G., and Chen, I.-M.: A flexure-based electromagnetic nanopositioning actuator with predictable and re-configurable open-loop positioning resolution, Precis. Eng., 40, 249-260, 2015.

Yu, J., Hu, Y., and Bi, S.: Kinematics feature analysis of a 3 DOF in-parallel compliant mechanism for micro manipulation, Chin. J. Mech. Eng., 17, 127-131, 2004.

Yu, J., Li, S., Su, H.-J., and Culpepper, M. L.: Screw Theory Based Methodology for the Deterministic Type Synthesis of Flexure Mechanisms, Journal of Mechanisms and Robotics, 3, 031008, doi:10.1115/1.4004123, 2011.

Zettl, B., Szyszkowski, W., and Zhang, W. J.: Accurate low DOF modeling of a planar complaint mechanism with flexure hinges: the equivalent beam methodology, Precis. Eng., 29, 237-245, 2004. 